\title{
ESTUDIO DEL MANEJO, CLASIFICACIÓN Y RECOLECCIÓN DE FITOSANITARIOS EN EL CULTIVO DE FRÍJOL (Phaseolus vulgaris) EN SIBUNDOY PUTUMAYO (COLOMBIA)
}

\author{
MANAGEMENT, CLASSIFICATION AND COLLECTION STUDY \\ OF PHYTOSANITAIRES IN THE BEANS CROP (Phaseolus \\ vulgaris) IN SIBUNDOY PUTUMAYO (COLOMBIA)
}

\begin{abstract}
${ }^{1}$ Adriana del Socorro Guerra Acosta, ${ }^{2}$ Anderson Alexander Mena Pantoja, ${ }^{3}$ Melaly Estefany Burbano Montenegro, 4 María Fernanda Burbano Montenegro, ${ }^{5}$ Lizeth María Pardo Potosí

${ }^{1}$ Ingeniera Agrónoma, Especialista en Gerencia Ambiental, Magister en Ciencias Agrarias, Instituto Tecnológico del Putumayo docente Titular tiempo completo, Sibundoy Putumayo, Colombia.

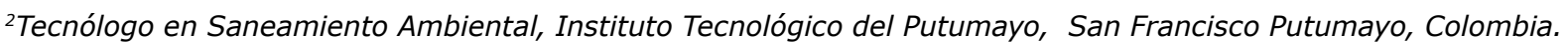

3,4,5Tecnóloga en Saneamiento Ambiental, Instituto Tecnológico del Putumayo, Sibundoy Putumayo, Colombia.

${ }^{1}$ aguerra@itp.edu.co ${ }^{2}$ aamena17s@itp.edu.co ${ }^{3}$ meburbano17s@itp.edu.co

${ }^{4} \mathrm{mfburbano17s@itp.edu.co}{ }^{5}$ Impardo17s@itp.edu.co
\end{abstract}

\section{RESUMEN}

Contextualización: El control de plagas, enfermedades, malezas en la producción de fríjol, uno de los cultivos más representativos a nivel económico en el municipio de Sibundoy, se caracteriza por el uso de agroquímicos. La problemática que estos generan se debe abordar desde su origen, manejo y disposición final de residuos post-consumo.

Vacío de investigación: El manejo de productos y residuos fitosanitarios en la producción agrícola ha generado problemas ambientales y a la salud humana a través del tiempo. El uso, almacenamiento, tipo de productos, disposición final de residuos y seguridad en la aplicación, realizados por los productores agrícolas es un tema poco evaluado en la región. Es por ello conveniente que se estudie la situación actual de esta problemática, que permite tomar decisiones y medidas apropiadas de manejo de este tipo de productos en los sistemas agrícolas.

Propósito del estudio: El objetivo de la investigación fue estudiar el manejo, clasificación y recolección de fitosanitarios en el cultivo de fríjol (Phaseolus vulgaris) en Sibundoy Putumayo Colombia. 
Metodología: Fue descriptiva, basada en la recolección de información primaria y secundaria, que permitió caracterizar diferentes aspectos, de manera cualitativa y cuantitativa. El análisis se realizó en dos fases. La primera fue la observación directa y aplicación de encuestas en campo, identificación de la manipulación, disposición final de residuos y percepción del riesgo. La fase dos, incluyo la recolección y clasificación de los recipientes de agroquímicos empleados por los agricultores durante 6 meses, la clasificación de los recipientes se realizó de acuerdo a los siguientes parámetros: nombre comercial, componente activo y categoría toxicológica. Además, se llevó a cabo el pesaje de los residuos generados en esta actividad agrícola. La información recolectada fue insumo importante para la realización de un análisis de correspondencia múltiple. La investigación diagnosticó el manejo que los agricultores le dan a los agrotóxicos durante el ciclo productivo de cultivo que son 6 meses, donde se identificó el uso inadecuado desde su recepción, manejo y disposición final.

Resultados y conclusiones: Se recolectaron 554 recipientes en total, 35\% corresponden a insecticidas, $25 \%$ fungicidas, $20 \%$ herbicidas y $18 \%$ sin identificación. El total de agricultores a quienes se les realizó la encuesta fueron 51 personas, el $43 \%$ de ellos recolectan los envases. En el análisis de conglomerados se obtuvo IV Clúster donde se agrupan los datos de mayor relevancia. Finalmente se concluye que el uso de agroquímicos respecto a su aplicación y disposición final se caracteriza por su inadecuado manejo, en aspectos como la dosificación, almacenamiento, seguridad de los operarios, el cual amenaza el equilibrio de los ecosistemas y la salud de los agricultores.

Palabras clave. Agrotóxico, envase, fitosanitario, sistema agrícola, recolección.

\section{ABSTRACT}

Contextualization: The pests, diseases and weeds control in the bean crop production, is one of the most representative crop at the economic level in the Sibundoy municipality, it is characterized by the use of agrochemicals. The problems that these generate must be addressed from its origin, management and final disposal of post-consumer waste.

Knowledge gap: The phytosanitary products and residues management in agricultural production has generated environmental and human health problems over the time. The use, storage, type of products, residues final disposal and safety in the application carried out by agricultural producers, it is a little evaluated subject in the region. Then, it is advisable to study the current situation of this problem, which allows taking decisions and proper handling measures of this type of product in the agricultural systems.

Purpose: The research objective was to study the management, classification and collection of phytosanitary products in the beans cultivation (Phaseolus vulgaris) in Sibundoy Putumayo Colombia.

Methodology: It was descriptive, based on the collection of primary and secondary information, which allowed the different aspects characterization, qualitatively and quantitatively. The analysis was carried out in two phases. The first one was direct observation and field surveys application, handling identification, waste final disposal and risk perception. The phase two included the agrochemical containers collection and classification used by farmers for 6 months. The containers classification was carried out according to the following parameters: trade name, active component and toxicological 
category. In addition, it was carried out the waste weighing generated in this agricultural activity. The information collected was an important input for conducting a multiple correspondence analysis. The investigation diagnosed the handling that farmers give to pesticides during the cultivation productive cycle, which is 6 months, where it was identified the inappropriate use from its reception, handling and final disposal.

Results and conclusions: In total, they were collected 554 containers, 35\% correspond to insecticides, $25 \%$ fungicides, $20 \%$ herbicides and $18 \%$ without identification.
The farmers total who were surveyed were 51 people, $43 \%$ of them collect the containers. In the conglomerate analysis, it was obtained Cluster IV where are grouped the most relevant data. Finally, it is concluded that the agrochemicals use with respect to their application and final disposal is characterized by their inadequate management, in aspects such as dosage, storage, and operator safety, which threatens the ecosystems balance and the farmers health.

Keywords. Agrotoxic, container, phytosanitary, agricultural system, harvesting.

\section{RESUMEN GRÁFICO}

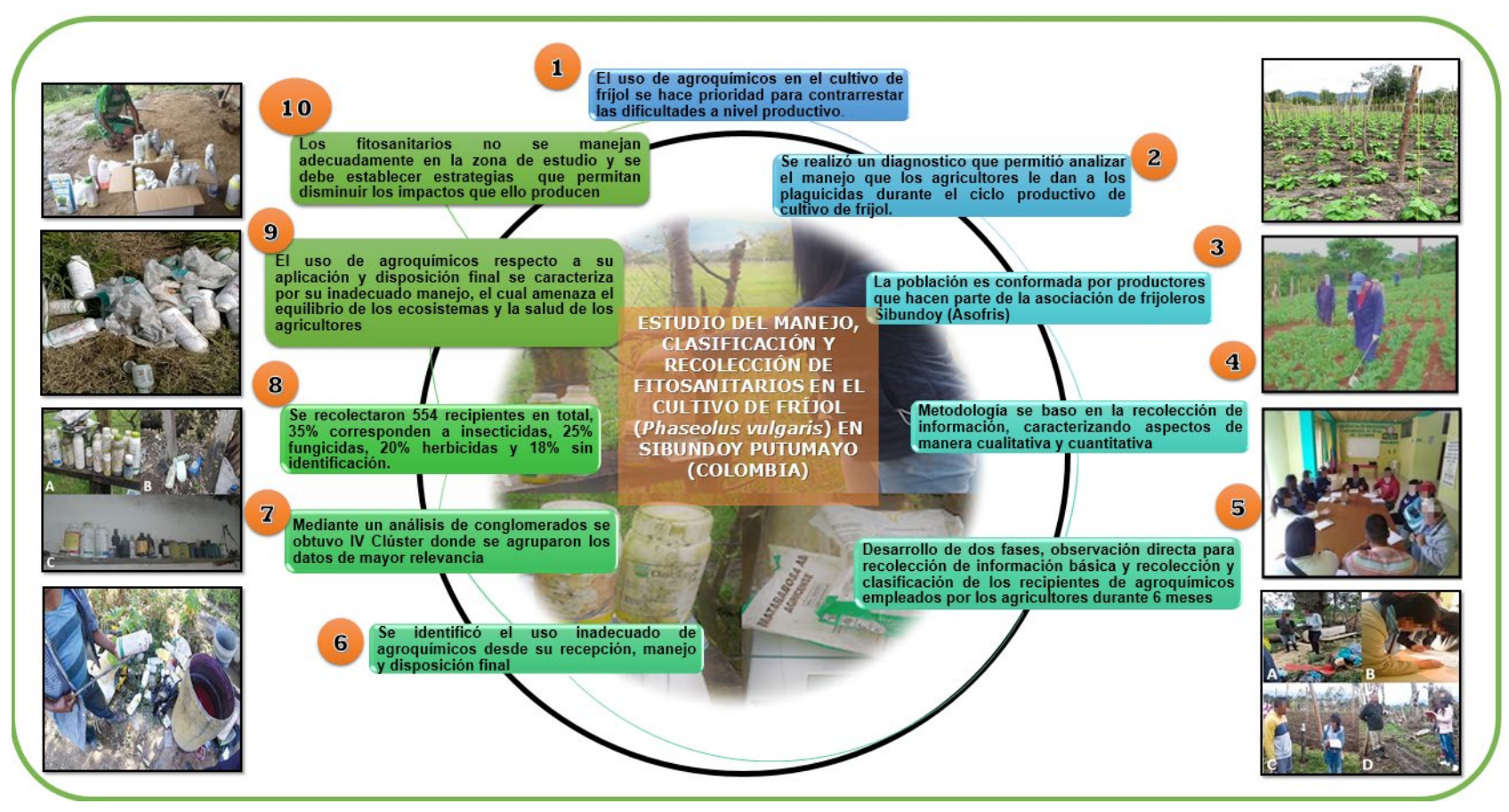




\section{INTRODUCCIÓN}

Los agroquímicos han contribuido en gran medida al aumento de los rendimientos en la agricultura mediante el control de plagas y enfermedades (Rekha, Naik, y Prasad, 2006). Las plagas pueden llegar a ocasionar hasta un $45 \%$ de pérdidas de producción anual de alimentos (Abhilash y Singh, 2008). Con frecuencia, el control químico es el único medio disponible y eficiente para atacar este problema por las altas poblaciones alcanzadas, por los artrópodos y los daños que estos ocasionan.

Estos productos han generado problemas de diversa índole derivados casi siempre de su uso inadecuado. Si en un principio se consideraron como la solución de los problemas fitosanitarios, actualmente la experiencia y el mejor conocimiento de la complejidad de los ecosistemas agrícolas, han demostrado que son un componente más de manejo inadecuado de plagas Pretty y Waibel, 2005). Debido al incremento en las dosis de agroquímicos y a su manejo inadecuado, se presenta acumulación de residuos en diversos ecosistemas, problemas en la salud humana, daños al medio ambiente, resistencia de los insectos a insecticidas y resurgencia de plagas, incremento de plagas secundarias y disminución de enemigos naturales (Harris, 2000).

Otro problema que se presenta por la mala utilización de fitosanitario es la falta de inocuidad de los productos agrícolas. La exposición a plaguicidas provoca una gama de problemas, cuyos peligros varían de acuerdo con el grado de exposición y manejo de los agroquímicos (Abhilash y Singh, 2008)
La agricultura desempeña un papel crucial en la economía del municipio de Sibundoy. El frijol predomina en la región y es la columna vertebral del sistema económico, destacándose por un manejo convencional en todo el proceso productivo (Levantamiento de suelos, capacidad de uso de las tierras y cobertura terrestre escala 1:25.000).

El objetivo de la investigación fue realizar un estudio del uso de fitosanitarios en el cultivo de fríjol del municipio de Sibundoy Putumayo Colombia, durante el periodo del cultivo 6 meses. Esta actividad permitió conocer la disposición de los envases de agroquímicos generados por los agricultores en los sistemas agrícolas evaluados, identificar mecanismos en el proceso de almacenamiento y aplicación de productos agroquímicos, además caracterizar toxicológicamente los productos usados.

\section{MATERIALES Y MÉTODOS Localización}

La investigación se realizó en las veredas Villa Fátima, Machindinoy, Tamabioy, El Ejido, Sagrado Corazón de Jesús y Campo Alegre del municipio de Sibundoy departamento del Putumayo (Figura 1), la temperatura media multianual es $16{ }^{\circ} \mathrm{C}$, altitud de la cabecera municipal es de $2100 \mathrm{~m}$, la humedad relativa es del $83 \%$ y precipitación promedio anual de 1578 mm (Corpoamazonía, 2010). El número de agrosistemas evaluados fue 51 que correspondió al total de productores encuestados, sin repetir predios por productor, la evaluación se realizó entre los meses de junio a diciembre semestre donde se produce el cultivo en la zona de estudio. 


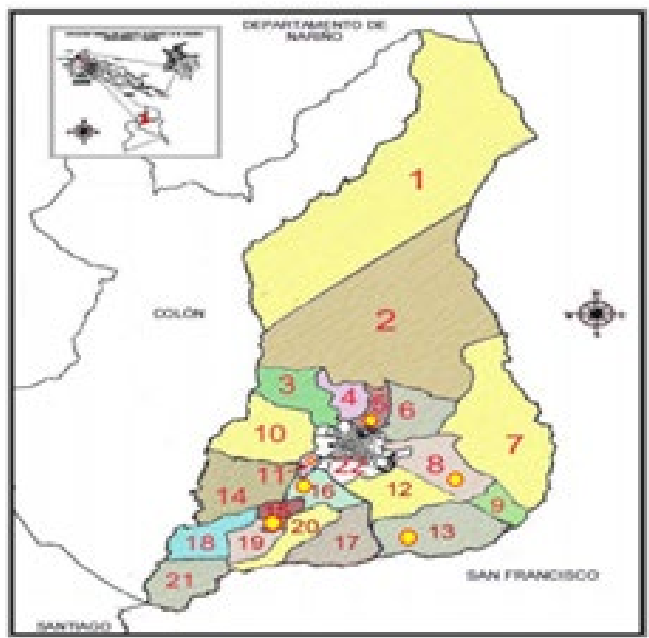

VEREDAS

No. 5 Vereda Campo Alegre

No. 8 Vereda Villa Fátima

No. 11 Vereda Machindinoy

No. 13 Vereda Tamabioy

No. 15 Vereda El Ejido

No. 16 Vereda Sagrado corazón

Figura 1. Ubicación del área de estudio en las veredas del municipio de Sibundoy.

Fuente: Plan de Gestión Ambiental Sibundoy Putumayo (2008).

\section{Población}

Formada por 59 productores de cultivo de que hacen parte de la asociación de frijoleros de Sibundoy (Asofris).

\section{Muestra}

Se tomó una muestra representativa de 51 productores de frijol, mediante la aplicación de la ecuación 1, expuesta por Hidalgo y Argoty citado por ( Fernández, 2010).

$$
n=\frac{N \times Z_{a}{ }^{2} \times p \times q}{d^{2} \times(N-1)+Z_{a}{ }^{2} \times p \times q} \quad(\text { Ecuacion } 1)
$$

Donde:

$p \times q=0,25$ (que corresponde a la probabilidad de acierto y fracaso).

$e=5 \%$ (porcentaje de error estimado) (0.05)

$z=1,96$ (correspondiente a una confianza del $95 \%)$.

$\mathrm{N}=59$ número de agricultores de la población $\mathrm{n}=51$ total de productores encuestados y predios evaluados.

\section{Fases metodológicas}

Fase 1. Se realizó observación directa a través de visitas a los diferentes agrosistemas, las cuales permitieron determinar el almacenamiento de los agroquímicos, señalización y equipos usados para la aplicación, de igual manera en esta fase se realizaron encuestas a los agricultores (Figura 2), donde se evaluaron 5 variables:

- Uso de fitosanitarios: productos más utilizados, frecuencia, dosis, criterio de aplicación y asistencia técnica.

- Cultivos sembrados: área, plagas más frecuentes y alternativas de control.

- Aspecto socioeconómico del agricultor: edad, género, tenencia de tierra y hectáreas cultivadas.

- Condiciones de aplicación segura: persona responsable de las aplicaciones, lugar de la mezcla, equipo de protección, lectura de etiquetas, conocimiento del nivel de toxicidad, manejo de envases vacíos y sobrantes de productos y mantenimiento de equipo de protección.

- Condiciones ambientales y percepción del riesgo: Usos de fuentes de agua, cambios existentes en los recursos naturales, conocimiento de síntomas de intoxicación. 


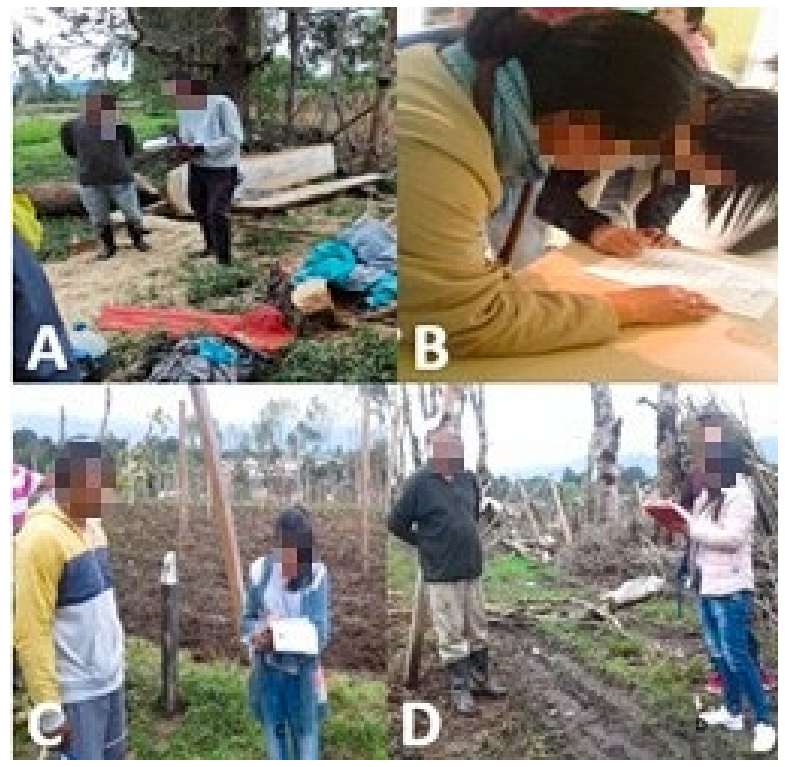

Figura 2. Visita a los sistemas de producción y aplicación de encuestas a los agricultores. A y B. Aplicación de encuestas a los agricultores C y D. Visitas a sistemas agrícolas.

Fuente: autores

Fase 2. Se realizó jornadas de recolección ingrediente activo, tipo de formulación y cay clasificación de envases y bolsas vacías de tegoría toxicológica, también se llevó a cabo agroquímicos utilizados por los agricultores, el pesaje de estos el cual permitió conocer durante un periodo de seis meses, (Figura 3) la cantidad en kilogramos de residuos que se teniendo en cuenta el tipo, nombre comercial, generan (Figura 4).

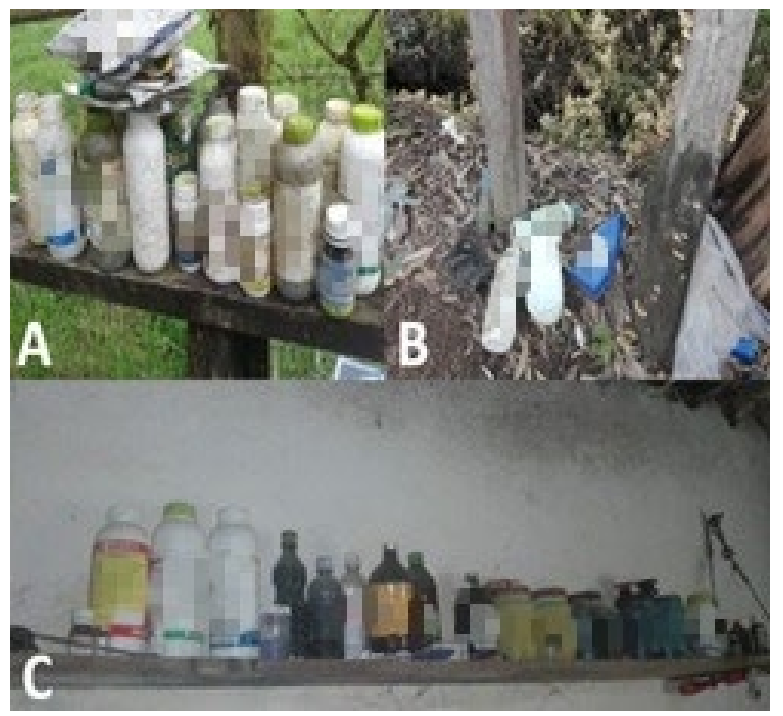

Figura 3. Recolección y clasificación de envases y papeletas vacías. A. Identificación de agroquímicos y papeletas B. Recolección de agroquímicos C. Clasificación de agroquímicos.

Fuente: autores 


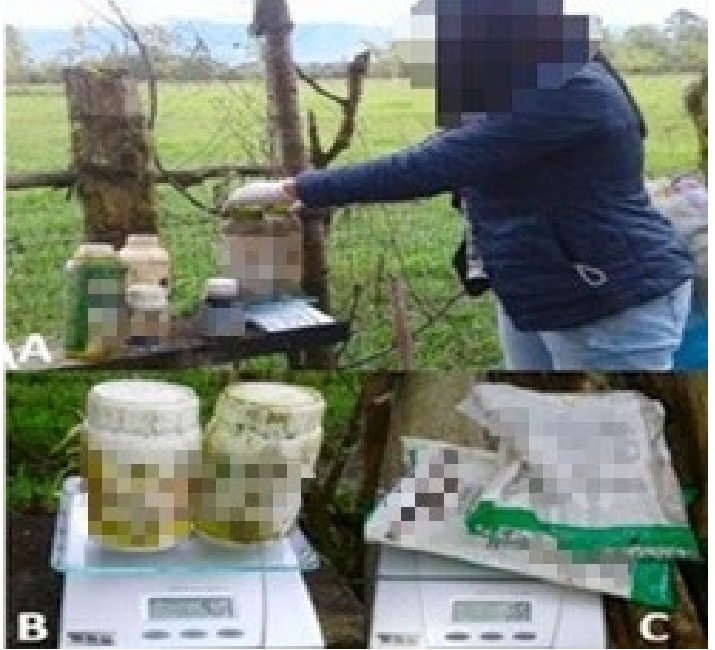

Figura 4. Pesaje de residuos de agroquímicos en los sistemas agrícolas. A y B. Pesaje de envases de agroquímicos y papeletas vacíos

C. Pesaje de papeletas vacías.

Fuente: autores

\section{Análisis estadístico}

La información obtenida en las encuestas fue sometida a un análisis multivariado de correspondencia múltiple Système Portable pour I'Analyse de Données (software usado fue SPAD V 5.0), el cual permite implementar una estrategia de análisis adecuada al tratamiento exploratorio multivariante de grandes tablas de datos. El análisis correspondencia es un modelo aleatorio, ya que el investigador no tiene control experimental sobre los individuos. El trabajo es eminentemente de tipo descriptivo y el análisis estadístico que se realiza para casos como éste, es un análisis multivariado y que por tratarse de variables categóricas (no cuantitativas), se utilizó una técnica multivariada factorial, el ACM (análisis de correspondencias múltiples), que permite analizar las relaciones entre las categorías de variables cualitativas a partir de una muestra de $n$ individuos, es una técnica estadística multivariante que busca agrupar elementos (o variables) tratando de lograr la máxima homogeneidad en cada grupo y la mayor diferencia entre los grupos. Uno de los objetivos de la investigación, se basó en la aplicación de encuestas realizadas a diferentes agricultores en veredas del municipio de Sibundoy y por tanto su análisis corresponde a un modelo aleatorio, ya que el investigador no tiene control experimental sobre los individuos.

Se realizó un análisis de conglomerados que permitió establecer los principales factores y el porcentaje de participación que tienen cada una de las variables evaluadas, facilitando realizar las correlaciones más pertinentes dentro de las preguntas planteadas en la encuesta.

Para visualizar mejor los resultados del análisis de conglomerados, con relación a las preguntas planteadas en la encuesta, se elaboró un dendograma. Esta una herramienta visual que puede ayudar a determinar el número de grupos que podrían representar mejor la estructura de los datos teniendo en cuenta la forma en que se van anidando los cluster y la medida de similitud a la cual lo hacen.

\section{RESULTADOS Y DISCUSIÓN Pesaje de residuos}

De acuerdo con los datos obtenidos (Tabla 1) en la recolección y clasificación de los residuos de agroquímicos usados en el cultivo de fríjol, se recolectaron los siguientes. 
Tabla 1. Productos identificados en la recolección y clasificación de residuos de agroquímicos

\begin{tabular}{l|c|c|c|c}
\hline PRODUCTO & TOTAL ENVASES & \% USOS EN LA ZONA & PESO kg & $\%$ PESO \\
\hline Insecticidas & 196 & $35,38 \%$ & 27,2 & $35 \%$ \\
\hline Fungicidas & 140 & $25,27 \%$ & 19,7 & $25 \%$ \\
\hline Herbicidas & 115 & $20,76 \%$ & 16,3 & $21 \%$ \\
\hline Sin etiqueta & 103 & $18,59 \%$ & 14,5 & $19 \%$ \\
\hline TOTAL & 554 & $100 \%$ & 77,7 & $100 \%$ \\
\hline
\end{tabular}

Fuente: Autores

\section{Caracterización toxicológica}

Tabla 2. Ingredientes activos de insecticidas usados con mayor frecuencia en el cultivo de fríjol

\begin{tabular}{|c|c|c|c|c|}
\hline \multicolumn{5}{|c|}{ INSECTICIDAS } \\
\hline $\begin{array}{l}\text { INGREDIENTE } \\
\text { ACTIVO }\end{array}$ & NOMBRE COMERCIAL & $\begin{array}{c}N^{\circ} \\
\text { ENVASES }\end{array}$ & $\begin{array}{c}\% \\
\text { USO }\end{array}$ & CATEGORÍA TOXICOLÓGICA \\
\hline Clorpirifos etil & Lorsban & 31 & 15,81 & III Medianamente Tóxico \\
\hline Cipermetrina & Cipertox & 27 & 13,77 & III Medianamente Tóxico \\
\hline Profenofos & Curacron & 24 & 12,24 & III Medianamente Tóxico \\
\hline Chlorpyrifos & Pyrinex & 21 & 10,71 & III Medianamente Tóxico \\
\hline Imidacloprid & Invicto & 18 & 9,18 & III Medianamente Tóxico \\
\hline Landacialotrina & Cihalotrina & 16 & 8,16 & III Medianamente Tóxico \\
\hline Lufenuron & Match & 16 & 8,16 & III Medianamente Tóxico \\
\hline Abacmetina & Abamec & 14 & 7,14 & III Medianamente Tóxico \\
\hline Fipronil & Bemol & 11 & 5,61 & III Medianamente Tóxico \\
\hline Diflubenzoron & Dimilin & 9 & 4,59 & IV Ligeramente Tóxico \\
\hline Clorpirifos & Agromil & 9 & 4,59 & III Medianamente Tóxico \\
\hline
\end{tabular}

Fuente: Autores, ficha técnica productos.

La totalidad de los agricultores de fríjol utiliza en un $35.38 \%$ insecticidas en su paquete de insumos. Los productores aplican 11 ingredientes activos diferentes por ciclo de cultivo según Tabla 2. Los insecticidas de mayor uso por cultivo son clorpirifos etil con $15,81 \%$, seguido por la Cipermetrina con $13,77 \%$ y Profenofos con $12,24 \%$ del total. De acuerdo con los resultados obtenidos en la tabla 2 se puede observar que la categoría toxicológica III de color azul moderadamente toxica es la que predomina en el uso los insecticidas con un $95.37 \%$.

En este contexto, las consecuencias ambientales del uso de insecticidas causan gran preocupación. Por lo general tienen un mayor 
impacto en el medio ambiente, los insecticidas se encuentran entre las herramientas agrícolas que están más asociadas con el daño ambiental. Su objetivo específico es eliminar plagas de insectos y por consecuencia puede que tenga un impacto letal o subletal en organismos que no son su objetivo (por ejemplo, recicladores de nutrientes del suelo, polinizadores de plantas y depredadores de plagas) y contaminar los alimentos producidos para los niveles tróficos superiores (Gregor J., Dominique y Ogusuku, 2008).

Tabla 3. Ingredientes activos de fungicidas usados con mayor frecuencia en el cultivo de fríjol

\begin{tabular}{|c|c|c|c|c|}
\hline \multicolumn{5}{|c|}{ FUNGICIDAS } \\
\hline INGREDIENTE ACTIVO & NOMBRE COMERCIAL & $\begin{array}{c}N^{\circ} \\
\text { ENVASES }\end{array}$ & $\begin{array}{c}\% \\
\text { USO }\end{array}$ & CATEGORÍA TOXICOLÓGICA \\
\hline Propineb & Antracol & 28 & 20 & III Medianamente Tóxico \\
\hline Chlorothalonil & Daconil & 25 & 17,85 & IV Ligeramente Tóxico \\
\hline Difenoconazole & Score & 22 & 15,71 & III Medianamente Tóxico \\
\hline Carbendazim & Carbendazim & 18 & 12,85 & IV Ligeramente Tóxico \\
\hline Mancozeb & Dithane & 18 & 12,85 & IV Ligeramente Tóxico \\
\hline Benomil & Benomyl & 16 & 11,42 & III Medianamente Tóxico \\
\hline Azoxystrobin & Amistar Top & 13 & 9,28 & II Altamente Tóxico \\
\hline
\end{tabular}

Fuente: Autores, ficha técnica productos.

La Tabla 3 incluye la cantidad de fungicidas utilizados con 7 ingredientes activos diferentes, además en cada fumigación realizan mezclas con un rango entre 11 a 26 veces en un ciclo de producción que dura aproximadamente 6 meses, esto depende de las condiciones ambientales, el manejo agronómico y el precio de demanda en el momento cercano a la cosecha.

Los fungicidas son compuestos que se utilizan para prevenir, mitigar, repeler o controlar alguna enfermedad en los cultivos. La principal fuente de contaminación al ambiente por el uso de estos es el residuo que resulta de su aplicación. Aunque su aplicación se restinga a zonas determinadas, su dispersión es universal. Se han encontrado restos de plaguicidas incluso en locaciones tan remotas como la Antártida. Cuando son liberados permanecen inalterados por largo tiempo en el ambiente. Como son sustancias poco solubles en agua, se evaporan pasando al aire o uniéndose a las partículas del suelo, como vapor o polvo. Pueden ser transportados grandes distancias y nuevamente ser depositados a través de lluvias sobre la tierra o aguas superficiales alterando la vida de muchos organismos ( Anguiano, y otros, 2011). 
Tabla 4. Ingredientes activos de herbicidas usados con mayor frecuencia en el cultivo.

\begin{tabular}{l|l|c|c|c}
\hline \multicolumn{4}{c}{ HERBICIDAS } \\
\hline $\begin{array}{l}\text { INGREDIENTE } \\
\text { ACTIVO }\end{array}$ & NOMBRE COMERCIAL & $\begin{array}{c}\text { No } \\
\text { ENVASES }\end{array}$ & $\%$ USO & CATEGORÍA TOXICOLóGICA \\
\hline Paraquat & Gramoxone & 28 & 24,34 & I Extremadamente Tóxico \\
\hline Metsulfuron Methyl & Metsulfuron & 25 & 21,73 & III Medianamente Tóxico \\
\hline Glifosato & Glifosato & 19 & 16,52 & IV Ligeramente Tóxico \\
\hline Fomesafen & Flex & 16 & 13,91 & III Medianamente Tóxico \\
\hline Diurom & Diurex & 11 & 9,56 & III Medianamente Tóxico \\
\hline Clomazone 48 EC & Clomazone & 9 & 7,82 & II Altamente Tóxico \\
\hline 2.4 D & Aminas Agrogen & 7 & 6,08 & III Medianamente Tóxico \\
\hline
\end{tabular}

Fuente: Autores, ficha técnica de productos.

Según la Tabla 4 los herbicidas de mayor uso ponderado por cultivo son principalmente Paraquat con un $24,34 \%$ el cual se aplica para desecar las plantas antes de la cosecha; con características de alta toxicidad según la banda de categoría toxicológica. Metsulfuron Methyl con $22 \%$ y Glifosato $16,52 \%$ con para preparar el terreno antes de la siembra. Este agroquímico afecta principalmente al recurso suelo, alterando especialmente el componente orgánico (Del Puerto, Suárez y Estrada, 2014).

En los resultados obtenidos con respecto al ingrediente activo se encontró que en la zona los agricultores hacen uso de 25 tipos de agroquímicos, de los cuales 11 son de acción insecticida, siete de acción fungicida y herbicida Tablas 2, 3 y 4 . Estos agroquímicos se clasificaron entre las categorías toxicológicas definidas en el decreto 1843 en su artículo 14, como I Extremadamente tóxico, II altamente tóxico, III medianamente tóxico y IV ligeramente tóxico.

Con respeto a la categoría toxicológica, la mayoría de agroquímicos utilizados corresponde a categoría III color azul, medianamente tóxico; las categorías II y IV tienen igual relevancia donde la primera es altamente tóxico al cual se le debe tener mucho cuidado respecto a la aplicación y dosificación que indica un riesgo alto y se encuentran en su mayoría en el grupo de los insecticidas y el segundo ligeramente toxico siendo el menos peligroso de acuerdo a la banda de colores se identifica con el color verde; cabe mencionar que en el grupo de los herbicidas se encuentra el único agroquímico (Paraquat) color rojo extremadamente toxico categoría I el cual debe reemplazarse por productos más seguros y que sean compatibles con programas de manejo integrado.

Los agroquímicos se clasifican y etiquetan según su grado de toxicidad en cuatro categorías: I, II, III y IV. A su vez, clasifica las fincas según el uso de estos insumos: de alto riesgo, aquellas que están al menos en I y II y su frecuencia de aplicación es igual o mayor a dos por mes; como fincas de bajo uso de insumos están aquellas donde solamente se aplican agroquímicos de las clases III y IV y que la frecuencia de aplicación no excede una al mes (Cruz, Trejos, Ser y Giraldo, 2011).

Los agroquímicos se dispersan en el ambiente y se convierten en contaminantes para los sistemas biótico (animales y plantas 
principalmente) y abiótico (suelo, aire y agua) amenazando su estabilidad. Las condiciones geomorfológicas de los suelos y las condiciones hidrogeológicas y meteorológicas de las zonas definen la ruta que siguen los mismos en el ambiente. La gran variedad de agroquímicos usados es alta y por consiguiente se hace un mal manejo siendo aplicados en dosis inadecuadas, lo que ha generado inestabilidad en los recursos naturales (Guerrero, 1998).

Cuando los agroquímicos ingresan en las cadenas alimentarias se distribuyen a través de ellas se concentran en cada nicho ecológico y se acumulan sucesivamente hasta que alcanzan una concentración letal para algún organismo constituyente de la cadena, o bien hasta que llegan a niveles superiores de la red trófica (Del Puerto, Suárez y Estrada, 2014).

\section{Análisis de resultados de la encuesta} Aspectos socioeconómicos de los agricultores. El $100 \%$ de los agricultores pertenece a la asociación de frijoleros Asofris. Con respecto al género de los productores el $96 \%$ son hombres y $4 \%$ son mujeres, de los cuales el $44 \%$ de los agricultores expuestos a plaguicidas son mayores de 40 años, el 26\% corresponde a edades entre 30 y 39 años y el $30 \%$ se encuentran en edades entre 23 y 29 años. Con relación a tenencia de tierra, el $65 \%$ de los productores cultiva en áreas arrendadas, $23 \%$ en usufructo y el $12 \%$ en áreas propias, el $53 \%$ de los productores manejan un área entre 1 y 2 hectáreas y el $47 \%$ de los productores manejan un área de 3 ha.

Condiciones de uso de agroquímicos. Las encuestas realizadas determinaron que el 94\% de los agricultores, han recibido asistencia técnica por parte de entidades sobre el uso de agroquímicos para su respectiva aplicación. En este sentido al ser un sistema intensivo de producción se recurre de manera constante a plaguicidas, por lo que el adecuado conocimiento de su uso y manejo es un requisito básico que constituye un elemento fundamental para orientar y estructurar estrategias que contribuyan a optimizar el uso de estos compuestos, sentando las bases para futuros estudios de evaluación de los efectos de estos productos en la salud humana y los ecosistemas (Ortega Martínez, y otros, 2014)

En la zona de estudio se encontró que para el $84 \%$ de los agricultores el tiempo donde dejan de aplicar productos agroquímicos es de 15 días antes de la cosecha, para algunos productos este periodo es el adecuado. Existen factores que presentan efectos más importantes en la estimación de un periodo de carencia y corresponden al tamaño de la planta al momento de aplicación, especie, técnica y zona agroecológica. La formulación del plaguicida, en general, no produce cambios significativos en la estimación del periodo de carencia, con excepción de algunas formulaciones que permiten la liberación controlada del ingrediente activo (Angioni, y otros, 2011)

La disposición final que los agricultores dan a los envases y bolsas vacías es, la recolección de estos residuos (43\%) lavado (35\%), los queman (18\%) los entierran (4\%) otros manejos. Algunas de estas acciones están relacionadas con lo que afirma Arellano (2005) en la que sugiere que enterrar los envases de plaguicidas, así como incinerarlos, es una disposición inadecuada que favorece el deterioro ambiental en las zonas agrícolas. La colocación y acumulación de residuos inutiliza las tierras para otros usos; además representa un riesgo para quienes viven cerca de los vertederos y acumulaciones de desechos, la acumulación indiscriminada de residuos puede convertir el agua en no apta para el consumo humano y el desarrollo de la vida acuática; la quema de residuos de agroquímicos ocasiona deterioro 
del aire juntamente con los gases tóxicos generados por la quema de residuos (Contreras Santos, 2008).

\section{Condiciones de una aplicación segura de} los agroquímicos. El $100 \%$ de los agricultores realizan la aplicación de los agroquímicos. El $84 \%$ de los productores consideran que es importante hacer uso de los elementos de protección personal, sin embargo únicamente el $16 \%$ hace uso de estos debido a que no los usan porque para ellos es incómodo, aclaran que la asociación a la que pertenecen hace dotación completa de los elementos de protección personal (EPP), entre estos están mascara, gafas, overol, guantes y botas; de igual manera capacita para su uso adecuado.

El elemento de protección personal es cualquier equipo o dispositivo destinado para ser utilizado por el trabajador, para protegerlo de los riesgos y aumentar su seguridad o su salud en el trabajo. Los EPP tienen como función principal proteger diferentes partes del cuerpo, para evitar que un trabajador tenga contacto directo con factores de riesgo que le pueden ocasionar una lesión o enfermedad (Flores, 2012).

La falta de conocimiento adecuado hace que no se haga un buen manejo de los agroquímicos, mencionan además que para la aplicación se rigen por los años de experiencia que llevan cultivando, es decir que no hacen una lectura de etiquetas antes de realizar la aplicación, llevando a dosificaciones excesivas e inadecuadas, la mayoría de agricultores desconocen el aspecto toxicológico, por ende no identifican la categoría toxicológica y su respectivo color de manera precisa desconociendo la información que esta puede brindar.
Aseguran que se brinda información pertinente para conocer la lectura de etiquetas, pero que en la mayoría de las veces no las toman en cuenta debido a que prefieren usar los insumos de acuerdo con la conveniencia del cultivo. Rother (2008) citado por (Arévalo, Bacca y Soto, 2013), afirma que es muy importante recordar que las etiquetas de los plaguicidas tienen un papel crucial en la protección de la salud y el medio ambiente.

El $84 \%$ de los agricultores guarda el producto sobrante en su empaque original, el sitio de almacenamiento es la casa de habitación, en donde destinan cuartos separados, se encontró que únicamente el $9 \%$ de los agricultores dispone los insumos en sus cultivos en una bodega exclusivamente para almacenar productos fitosanitarios.

Durante las visitas en campo se observó que en ocasiones se utilizan los mismos lugares de almacenamiento de agroquímicos para consumo de alimentos, lo que aumenta el riesgo de envenenamiento accidental para cualquiera de los individuos expuestos. Según (Santillán, 2017), menciona que los sitios de almacenamiento de plaguicidas deben estar aislados de viviendas y centros educacionales, hecho que no se cumple con los agricultores de la zona.

\section{Análisis de correspondencia múltiple (ACM)}

Con el Análisis de Correspondencia Múltiple $(A C M)$, según la tabla 5 , se pudo determinar que de los 26 factores, los once (11) primeros permiten explicar el $73.37 \%$ de la variabilidad total, indicando que el primer factor explica el $10.76 \%$, el segundo el $9.23 \%$, el tercero el $8.48 \%$, entre los tres factores se explica el $28.47 \%$ de la variabilidad de la encuesta aplicada a los productores encuestados. 
Tabla 5. Valores propios de las variables cuantitativas resultantes del ACM

\begin{tabular}{l|c|c|c}
\hline FACTOR & VALOR PROPIO & PORCENTAJE (\%) & PORCENTAJE ACUMULADO \\
\hline 1 & 0.2332 & 10.76 & 10.76 \\
\hline 2 & 0.2001 & 9.23 & 20.00 \\
\hline 3 & 0.1837 & 8.48 & 28.47 \\
\hline 4 & 0.1649 & 7.61 & 36.08 \\
\hline 5 & 0.1529 & 7.06 & 43.14 \\
\hline 6 & 0.1365 & 6.30 & 49.45 \\
\hline 7 & 0.1214 & 5.60 & 55.05 \\
\hline 8 & 0.1166 & 5.38 & 60.43 \\
\hline 9 & 0.0994 & 4.59 & 65.02 \\
\hline 11 & 0.0914 & 4.22 & 69.23 \\
\hline
\end{tabular}

Fuente: Autores

\section{Análisis de conglomerados de las encuestas.}

Tabla 6. Análisis de conglomerados

\begin{tabular}{l|c|c|c|c} 
Grupo & $\begin{array}{c}\text { No de } \\
\text { encuestas }\end{array}$ & \multicolumn{1}{c}{ Encuestas } & $22 \%$ \\
\hline I & 11 & $41,2,37,23,36,29,17,39,24,45,1$ & $35 \%$ \\
\hline II & 18 & $9,13,18,22,42,44,3,34,31,49,20,7,27,5,15,11,51,35$, & $29 \%$ \\
\hline III & 15 & $12,16,8,21,32,38,19,48,33,4,26,43,28,40,14$ & $14 \%$ \\
\hline IV & 7 & $50,6,47,25,10,30,46$ & \\
\hline
\end{tabular}

Fuente: Autores

De acuerdo con la tabla 6, y figura 5 en el grupo I se incluyen 11 encuestas que corresponde al $22 \%$, el grupo II 18 encuestas equivalentes al 35\%, el grupo III 15 encuestas con un
$29 \%$ finalmente en el grupo IV con 7 correspondiente al $14 \%$ de la totalidad de sistemas evaluados respectivamente. 


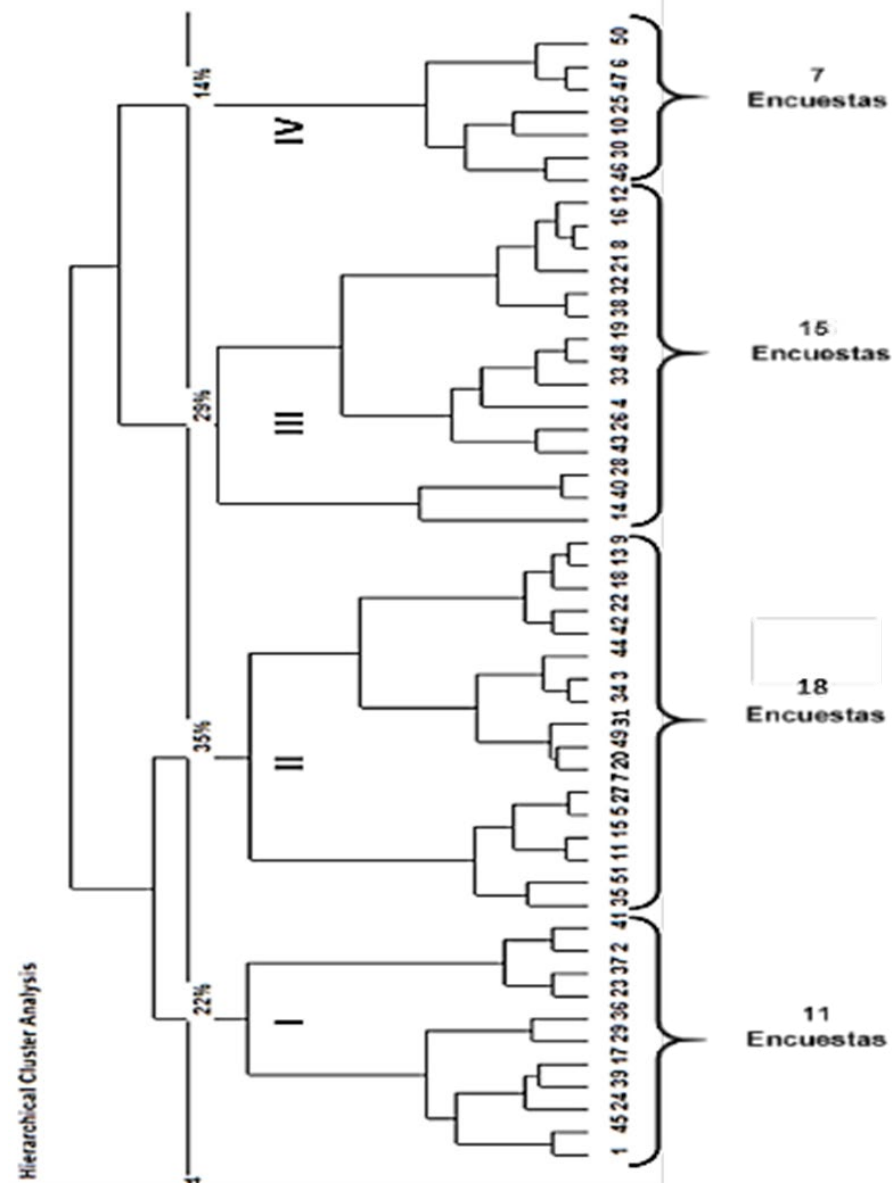

Figura 5. Dendrograma de clúster para encuestas realizadas en campo.

Fuente: Autores

\section{Análisis de clúster (grupo) jerárquico de encuestas}

\section{Clúster (Grupo) I/IV}

Tabla 7. Caracterización de variables grupo I

\begin{tabular}{|c|c|c|c|}
\hline Grupo & $N^{\circ}$ de encuestas & Encuestas & Porcentaje \\
\hline I & 11 & $41,2,37,23,36,29,17,39,24,45,1$ & $22 \%$ \\
\hline
\end{tabular}

Fuente: Autores

El grupo (I), incluye 11 encuestas cercana una de la otra y su aproximación al origen de las 51 aplicadas que corresponden al $22 \%$ del total, (Tabla 7). Este grupo está caracterizado porque el área dedicada al cultivo es menor a 2 hectáreas con un $72 \%$ de las personas a las que se les aplico la encuesta, además a la pregunta en qué lugar se almacenan los 
agroquímicos, los encuestados utilizan un estante, esta práctica es importante debido a que el lugar elegido para los plaguicidas lo cual es positivo ya que de esta manera se minimiza el contacto que puedan tener las personas y el medio ambiente con estos envases los cuales poseen una alta toxicidad, no debe estar cerca de cursos de agua, pozos y otras fuentes de suministro de agua para uso doméstico o para el ganado, ya que éstas podrían contaminarse a raíz de pérdidas o derrames que se produzcan en el almacén. Igualmente, otra variable de gran relevancia de este grupo en cuanto a la disposición de los envases de residuos fue la práctica del triple lavado.

De acuerdo con (Souza y Gebler, 2013), entre los principales determinantes del destino final de envases vacíos de plaguicidas se encuentra el tipo de material que los constituye y su peligrosidad para el medio ambiente, determinada en función de la posibilidad de llevar a cabo el proceso del triple lavado o triple enjuague en el momento de preparación de los solventes o soluciones indicadas. Según (Said Cometti y Gama Alves, 2010), si estos residuos son desechados en el medio ambiente, pueden contaminar el suelo y la capa freática.

Otro aspecto que tiene en cuenta que el grupo I es la presencia de plagas, donde el Boerhaavia difusa L (tostón), es el que predomina con $63 \%$, al respecto (Valencia, 2018), afirma que estas especies significan una verdadera amenaza para la producción agrícola competitiva y sostenible, porque no están respondiendo al control esperado de algunos plaguicidas químicos por igual. En consecuencia, hoy en día se acepta que ninguna alternativa de control individual sería suficiente para reducir la incidencia y el daño causado por las plagas. Por el contrario, se requiere la combinación e integración de diferentes medidas de control químico.

\section{Clúster (Grupo) II/IV}

Tabla 8. Caracterización de variables grupo II

\begin{tabular}{l|c|l|l|} 
Grupo & \multicolumn{1}{|c}{$N^{\circ}$ de encuestas } & \multicolumn{1}{|c}{ Encuestas } & \multicolumn{1}{c}{ Porcentaje } \\
\hline \multirow{2}{*}{18} & $9,13,18,22,42,44,3,34,31,49,20,7,27,5,15$, & $35 \%$ \\
\hline
\end{tabular}

Fuente: Autores

El grupo (II), se encuentra conformado por 18 encuestas de los 51 agricultores evaluados y que corresponden al 35\% del total siendo este el más significativo según tabla 8 .

Donde se agruparon aspectos como el área dedicada al cultivo entre 2 a 3 hectáreas, se identificó a diferencia del primer grupo este utiliza una bodega o caseta como almacenamiento de productos a utilizar. Además los agricultores de este grupo la tenencia de tierra es propia, en este aspecto (Padilla, Reyes, Lara, y Pérez,
2012), afirman que los apoyos y el incremento del precio que reciben los productores son una distorsión al aumentar el área sembrada del producto por arriba del equilibrio de mercado, e indirectamente se aumenta el uso de agua, agroquímicos y otros insumos.

La presencia de plagas en este grupo predomina con un $72 \%$ haciendo referencia al $\mathrm{Li}$ riomyza trifolii (minador). Según (Hidroponía, 2015), por la presencia de estas se disminuye la capacidad fotosintética, siendo un aspecto 
negativo para la parte ambiental. Existen distintas formas de erradicar esta plaga, pero principalmente los agricultores han optado por hacer uso de agroquímicos en altas dosis, incrementando los impactos a los ecosistemas donde hay diversidad de vidas de fauna y flora.

En este grupo la maleza que más ataca al cultivo es la Cynodon dactylon (gramilla) con $66 \%$ de los encuestados (Perrachon, 2010), afirma que para erradicarla es necesario una aplicación de glifosato que tiene como objetivo debilitar a la planta y a su vez acabar con los organismos benéficos dejando como consecuencia un desequilibrio en la estabilidad y fertilidad del suelo.
El fungicida más utilizado según al grupo (II) es el Daconil, afirmación que manifiestan el $78 \%$ de los encuestados, el cual según la etiqueta correspondiente menciona que dentro de los riesgos ambientales esta que es altamente toxico para especies acuáticas, acabando de manera inmediata con la micro y macrofauna por tal motivo se debe evitar que el producto entre en contacto con ambientes acuáticos. Además, menciona que es prácticamente no toxico para aves y virtualmente no toxico para abejas, pero que de una u otra manera puede alterar su forma de vida normal en un lapso determinado.

\section{Clúster (Grupo) III/IV}

Tabla 9. Caracterización de variables grupo III

\begin{tabular}{l|c|c|c|c|} 
Grupo & \multicolumn{2}{|c}{$N^{\circ}$ de encuestas } & Encuestas & Porcentaje \\
\hline III & 15 & $12,16,8,21,32,38,19,48,33,4,26,43,28,40,14$ & $29 \%$ \\
\hline
\end{tabular}

Fuente: Autores

Para el grupo (III) según tabla 9, el cual obtuvo 15 encuestas con $29 \%$, el área cultivada es mayor a 3 hectáreas que corresponde al $80 \%$ de los encuestados, los agricultores son arrendatarios, este grupo realiza actividades de recolección de residuos agroquímicos. Resaltando que a mayor número de hectáreas cultivas será más alto el uso de agroquímicos por ende los impactos a los ecosistemas se incrementaran.

Para el $46 \%$ de las personas encuestadas la babosa (Sarasinula plebeia), en la zona de estudio estas proliferan fácilmente debido a que la humedad ambiental es alta por encima del $85 \%$ y temperaturas medias son condiciones idóneas para su desarrollo.

Cabe mencionar que los agricultores indican que desde que hacen la preparación del suelo para la siembra al eliminar las malezas también aplican agroquímicos para acabar con la babosa. Pues cuando hay maleza la babosa hace más daño. Según (Avila, Conrado, Galeas y Argueta, 2005), se debe usar agroquímicos para controlar la babosa cuando la cantidad de esta plaga lo amerita, de lo contrario al hacer uso excesivo se puede acabar con la biodiversidad y su autorregulación al igual que con los depredadores naturales.

Frente a la presencia de enfermedades el $73 \%$ de los productores de este grupo manifiestan que Pythium es la enfermedad fungosa que más ataca a los cultivos en la zona de estudio, esta aparece y prolifera en cultivos con alta humedad, puede mantenerse activo durante muchos años en el sustrato del suelo una vez contaminado. 


\section{Clúster (Grupo) IV/IV}

Tabla 10. Caracterización de variables grupo IV

\begin{tabular}{|l|c|c|c|}
\hline Grupo & No de encuestas & \multicolumn{2}{c|}{ Encuestas } \\
\hline IV & 7 & $50,6,47,25,10,30,46$ & $14 \%$ \\
\hline
\end{tabular}

Fuente: Autores

Con respecto a las 7 encuestas que conforman el grupo (IV) el cual corresponde al $14 \%$ del total según tabla 10. Se encontró que este grupo de agricultores quema los envases de residuos agroquímicos, este manejo es inadecuado y puede causar daños ambientales a ecosistema cercanos, según (Diaz, Pagán, \& Silva, 2017), el destino correcto de los envases hasta un lugar adecuado evita que estos sean tirados al aire libre y contaminen el medio ambiente. Por lo tanto, hay beneficios de este tipo de logística inversa que proporcionan una mayor preservación del medio ambiente, beneficios para la sociedad y para las generaciones futuras, este autor propone la logística inversa que se aplica o consiste en la devolución de los envases vacíos de plaguicidas, como una contribución al medio ambiente, especialmente, mediante el reciclaje.

La presencia de malezas donde el $100 \%$ de los encuetados menciona que el Polygonun nepalense (corazón herido) es el que ataca con mayor frecuencia y cantidad.

El fungicida antracol es el más utilizado en los cultivos de este grupo esto lo manifiestan el $85 \%$ de los encuestados. Siendo estos el único grupo de sustancias químicas que se aplican deliberadamente en el medio ambiente, con el objeto de controlar hongos en plantas (Pesticide Action Network, 2010). Sin embargo, la mayoría de los fungicidas no actúan de manera específica sobre el hongo objetivo, ya que afectan simultáneamente a especies no objetivo, alterando el balance ecológico repetidas aplicaciones de productos fitosanitarios tienen consecuencias ambientales, tales como la reducción de la calidad del agua y del suelo, por presencia y acumulación de residuos, sumado al deterioro en la calidad del aire por volatilización de sustancias activas (Viglizzo, Esteban y Ernesto, 2010)

El lugar de almacenamiento el $85 \%$ los agricultores encuestados lo realizan en su vivienda, los agricultores y usuarios de plaguicidas deben tener en su finca un lugar adecuado para guardar sus envases lavados y perforados mientras hacen la devolución a la entidad encargada para dicho fin.

Los cuatro grupos de la representación se muestran en la figura 6 la que indica la distribución de las 51 encuestas realizadas a los agricultores sobre el uso y manejo de fitosanitarios, se puede determinar la relación que existen entre las variables predominantes en cada pregunta. 


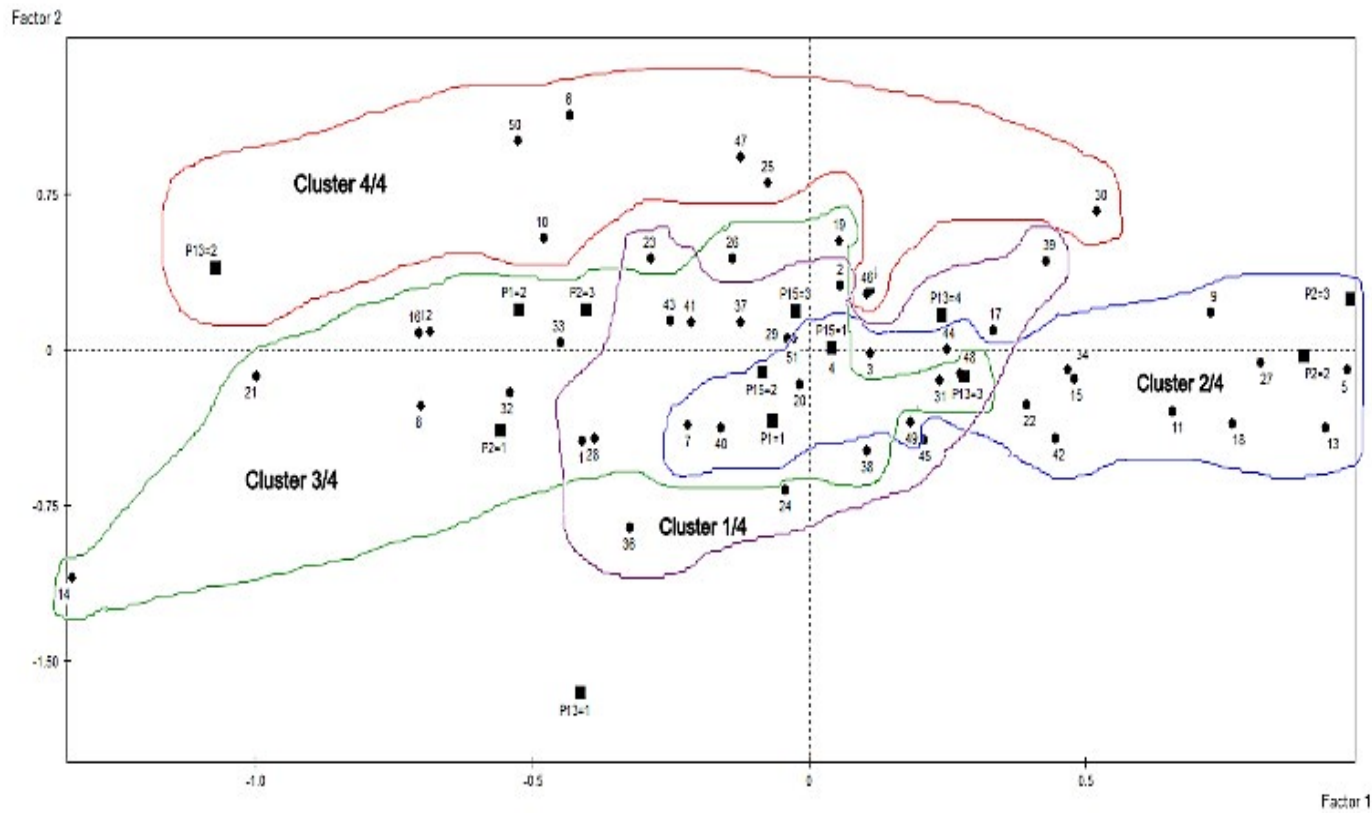

Figura 6. Representación gráfica de las variables y preguntas evaluadas en las encuestas sobre el uso y manejo de agroquímicos en el municipio de Sibundoy Putumayo.

Fuente: Autores

\section{CONCLUSIONES}

El uso de agroquímicos en la zona de estudio responde a un valor alto con una amplia variedad de 25 ingredientes activos diferentes. Destacándose el grupo de los insecticidas con 11 ingredientes activos. Posterior a estos están los fungicidas y herbicidas a los cuales se agrupan 7 ingredientes activos, con diferentes categorías toxicológicas donde predomina la III de color azul - moderadamente toxico.

El $43 \%$ de los agricultores con respecto a la disposición de los envases y papeletas de agroquímicos vacíos se hace mediante la entrega correspondiente a la empresa Colecta, de los cuales el $35 \%$ realiza el triple lavado, pero no hace la adecuada perforación, el $17 \%$ y $5 \%$ hacen una mala disposición de estos donde para el primer valor se queman y para el segundo se entierran lo cual representa un peligro ambiental.

Para la aplicación de los insumos agrícolas los productores de fríjol en su mayoría no hacen un manejo adecuado respecto a las dosis ya que en ocasiones prefieren sub dosificar a conveniencia del cultivo, además algunos no utilizan elementos de protección personal durante el momento de preparación de los diferentes productos ni mucho menos cuando hacen su aplicación.

Los agricultores carecen de conocimientos básicos en cuanto a los efectos ambientales que se pueden ocasionar por el manejo inadecuado de los agroquímicos por ende el $11 \%$ menciona que no se han presentado cambios en los recursos naturales, mientras que el $88 \%$ creen conveniente que las autoridades ambientales deben intervenir.

\section{RECOMENDACIONES}

Generar procesos de capacitación teórico práctico que permitan dar a conocer a los agricultores de la zona el uso adecuado de los agroquímicos con el fin de minimizar los impactos negativos al medio ambiente, y que a su vez enseñe la implementación de buenas 
prácticas agrícolas como factor positivo para los aspectos del desarrollo sostenible. Implementar estrategias encaminadas a mejorar la disposición final de residuos de envases y papeletas usados para la producción de frijol con el propósito de generar una cultura de cambio frente a este aspecto.

\section{CONTRIBUCIÓN DE LA AUTORÍA}

Primer autor: Metodología, investigación, análisis de datos, conceptualización, logística escritura - borrador original, edición. Segundo autor: Investigación, conceptualización, análisis de datos, diagramación, escritura. Tercer autor: Investigación, conceptualización, análisis de datos, diagramación, escritura. Cuarto autor: Investigación, conceptualización, análisis de datos, diagramación, escritura. Quinto autor: Investigación, conceptualización, análisis de datos, diagramación, escritura.

\section{AGRADECIMIENTOS}

Al Instituto Tecnológico del Putumayo, al Centro de Investigación y extensión Científica y Tecnológica CIECYT, al Dr Hernando Criollo Escobar (Q.E.P.D), docente de la Facultad de Ciencias Agrícolas de la Universidad de Nariño, por su apoyo en los procesos de la investigación, a los productores de fríjol del municipio de Sibundoy que se vincularon en el proyecto.

\section{LITERATURA CITADA}

Abhilash, y Singh, N. (2008). Pesticide use and application: An Indian Scenario. India: Publmed. (pág 7)

Angioni, A., Dedol, F., Garau, A., Sarai, G., Cabras, P., y Cabon, P. (2011). Part B: Pesticides, Food Contaminants, and Agricultural Wastes. En Journal of Environmental Science and Health, (pág. 8). United States: MIARMatriz de Información para el Análisis de Revistas.

Anguiano, O., Souza, M., Ferrari, A., Soleño, J., Pechen, A., y M, C. (4 de Junio de 2011). La Reserva. Obtenido de Los plaguicidas y sus efectos sobre el medio ambiente : http://www.lareserva. com/home/plaguicidas_pesticidas_efectos_medio_ambiente
Arévalo C., A., Bacca, T. \& Soto G., A. (2014). Diagnóstico del uso y manejo de plaguicidas en fincas productoras de cebolla junca Allium fistulosum en el municipio de Pasto Nariño. Revista Luna Azul, 38, 132-145. Recuperado de http://lunazul.ucaldas.edu.co/index.php?option $=$ content\&task $=\mathrm{v}$ iew\&id $=898$

Avila, F., Conrado, A., Galeas, W., y Argueta, R. (2005). Organización de las Naciones Unidas para la Agricultura y la Alimentaria. Obtenido de http://www.fao.org/3/a-at785s.pdf

Contreras Santos, M. J. (26 de Agosto de 2008). revistas.unal.edu.co. Obtenido de Universidad Nacional de Colombia:Santa Fé de Bogota Colombia https://revistas.unal.edu.co/index.php/tsocial/ article/view/14084/14844

Corporación para el desarrollo del sur de la Amazonia Corpoamazonia (Ed.). (2010). Plan de Ordenación y Manejo de la cuenca alta del río Putumayo. Mocoa: Corpoamazonía, WWF y Asociación Ampora, 130 p.

CRUZ C., G.; TREJOS P., J.F.; SERNA G. C.A.; CALDERÓN C., P.A. Evaluación de ingredientes activos de plaguicidas aplicados en sistemas de producción cafeteros certificados y no certificados en Cundinamarca y Santander Revista Cenicafé 62(1):17-31.2011 http://www.academia. edu/37059949/EVALUACI\%C3\%93.

Del Puerto, A., Suárez, S., y Estrada, D. (2014). Efectos de los plaguicidas sobre el ambiente y la salud. Revista Cubana de Higiene y Epidemiología. 2014;52 (3):372-387 http://www.scielo.sld. cu/pdf/hie/v52n3/hig10314.pdf

Diaz, M., Pagán, M., y Silva, S. (2017). Logística inversa de envases de plaguicidas: percepción de los pequeños y medianos productores agrícolas. Redalyc, 16.

Fernández, P. (01 de Diciembre de 2010). FIESTERRA. Obtenido de Determinación del tamaño muestral: https://www.fisterra.com/mbe/ investiga/9muestras/9muestras2.asp

Guerrero, Ricardo. (1998). Monómeros. Obtenido de Fertilización de cultivos en clima frío: Santafé de Bogotá. Colombia, Monómeros Colombo Venezolanos S.A. (E.M.A.) http://www.monomeros. com/descargas/dpmanualfrio.pdf

Harris, J. (2000). Chemical Pesticide Markets, Health Risks and Residues. Reino Unido: CABI. 
Flores, K. (2012). Importancia del uso de elementos de protección personal Karol Flores Ingeniera en prevención de riegos. https://karolfloresl. blogspot.com/2012/11/importancia-del-uso-deelementos-de.html

Gregor J.D., Dominique, E., y Ogusuku, E. (2008). Uso de insecticidas: contexto y consecuencias ecológicas. Rev Peru Med Exp Salud Pública. 2008; 25(1):74-100. http://www.scielo.org.pe/ $\mathrm{pdf} / \mathrm{rins} / \mathrm{v} 25 \mathrm{n} 1 / \mathrm{a} 11 \mathrm{v} 25 \mathrm{n} 1 . \mathrm{pdf}$

Hidroponía. (11 de Semptiembre de 2015). HIDROPONÍA. $m x$. Obtenido de HIDROPONÍA. mx- EI Blog de Hydro Environment: http://hidroponia. $\mathrm{mx} /$ como-afecta-a-los-cultivos-el-minador-dehoja/J

Ortega-Martinez LD, Martínez-Valenzuela C, Huerta-De la Peña A, Ocampo-Mendoza J, SandovalCastro E, Jaramillo-Villanueva JL (2014) Uso y manejo de plaguicidas en invernaderos de la región norte del estado de Puebla, México. Acta Universitaria 24: 3-12. http://www.acuedi.org/ ddata/8463.pdf

Padilla, L., Reyes, E., Lara, I., y Pérez, Ó. (2012). Competitividad, eficiencia e impacto ambiental de la producción de frijol (Phaseolus vulgaris L.) en Zacatecas, México. Scielo, 15.

Perrachon, Julio. (2010). Julio. Obtenido de ¿Cómo controlar la gramilla? Revista del Plan Agropecuario https://www.planagropecuario.org.uy/publicaciones/revista/R116/R116_38.pdf

Pesticide Action Network. (2010). Environmental effects of pesticide. EUROPE.

Pretty, \& Waibel. (2005). Paying the Price: The Full Cost of Pesticides. En: Pretty, J.N. (comp.), The Pesticide Detox: Towards a More Sustainable Agriculture. Londres.
Rekha, Naik, \& Prasad. (2006). Pesticide residue in organic and convetional food-risk. Journal of Chemical Healt and Safety. 13(6), 12-19. doi:10.1016/j.chs.2005.01.012 https://sci-hub. st/https://doi.org/10.1016/j.chs.2005.01.012

Santillán, Andrea. (2017). Dspace Escuela Superior Politécnica de Chimborazo. Obtenido de diagnóstico del uso y manejo de plaguicidas en cuatro comunidades del Cantón Guamote, provincia de Chimborazo. Riobamba Ecuador http://dspace. espoch.edu.ec/bitstream/123456789/6651/1/1 3T0843.pdf

Said Cometti, J. L., y Gama Alves, I. T. (07 de Mayo de 2010). Responsabilidad Post-Consumo y Logística Reversa: El Caso de los Embalajes de Agrotóxicos en Brasil. Sustentabilidadeem Debate.

Souza, V., y Gebler, L. (2013). Análise de cenário envolvendo embalagens. Ecotoxico e Medio Ambiente, 8. Pesticidas: Revista de ecotoxicologia e medio ambiente, ISSN 0103-7277, Vol. 23, 2013, págs. 75-82 https://dialnet.unirioja.es/servlet/ articulo?codigo $=5007021$

Valencia, E. (24 de Septiembre de 2018). Crop science Colombia. Obtenido de Las Súper Plagas agrícolas:https://www.cropscience.bayer.co/esCO/Centro-de Noticias/Noticias/2018/05/SuperPlagas.aspx

Viglizzo, Ernesto Fy Jobbágy Esteban (2010). Expansión de la frontera agropecuaria en Argentina y su impacto ecológico-ambiental. Argentina. https:// www.researchgate.net/publication/275345088 Expansion_de_La_Frontera_Agropecuaria_En Argentina_Y_Su_Impacto_Ecologico-Ambiental
Conflicto de Intereses Los autores declaran no tener ningún conflicto de intereses

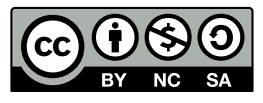

